\title{
AMENDMENTS
}

\section{Publisher Correction: BAF complex vulnerabilities in cancer demonstrated via structure-based PROTAC design}

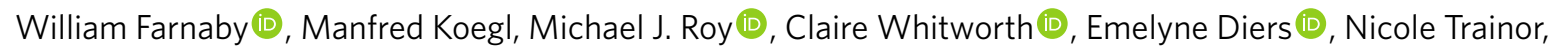
David Zollman, Steffen Steurer, Jale Karolyi-Oezguer, Carina Riedmueller, Teresa Gmaschitz, Johannes Wachter, Christian Dank, Michael Galant, Bernadette Sharps, Klaus Rumpel, Elisabeth Traxler, Thomas Gerstberger, Renate Schnitzer, Oliver Petermann, Peter Greb, Harald Weinstabl, Gerd Bader, Andreas Zoephel, Alexander Weiss-Puxbaum, Katharina Ehrenhöfer-Wölfer (D), Simon Wöhrle (D), Guido Boehmelt, Joerg Rinnenthal, Heribert Arnhof, Nicola Wiechens, Meng-Ying Wu, Tom Owen-Hughes, Peter Ettmayer (D), Mark Pearson, Darryl B. McConnell(D) and Alessio Ciulli (i)

Correction to: Nature Chemical Biology, https://doi.org/10.1038/s41589-019-0294-6, published online 10 June 2019

In the version of this article originally published, several lines of text in the last paragraph of the right column on page 1 of the PDF were transposed into the bottom paragraph of the left column. The affected text of the left column should read "The ATP-dependent activities of the BAF (SWI/SNF) chromatin remodeling complexes affect the positioning of nucleosomes on DNA and thereby many cellular processes related to chromatin structure, including transcription, DNA repair and decatenation of chromosomes during mitosis ${ }^{12,13}$." The affected text of the right column should read "SMARCA2/4 ${ }^{\mathrm{BD}}$ inhibitors are thus precluded from use for the treatment of SMARCA4 mutant cancers but could provide attractive ligands for PROTAC conjugation. Small molecules binding to other bromodomains have been successfully converted into PROTACs by conjugating them with structures capable of binding to the E3 ligases von Hippel-Lindau (VHL) or cereblon ${ }^{5,6,10,11,25,26,27}$." The errors have been corrected in the PDF version of the paper.

\section{Addendum: A cellular chemical probe targeting the chromodomains of Polycomb repressive complex 1}

Jacob I Stuckey, Bradley M Dickson, Nancy Cheng, Yanli Liu, Jacqueline L Norris, Stephanie H Cholensky, Wolfram Tempel, Su Qin, Katherine G Huber, Cari Sagum, Karynne Black, Fengling Li, Xi-Ping Huang, Bryan L Roth, Brandi M Baughman, Guillermo Senisterra, Samantha G Pattenden, Masoud Vedadi, Peter J Brown, Mark TBedford, Jinrong Min, Cheryl H Arrowsmith, Lindsey I James and Stephen V Frye

Addendum to: Nat. Chem. Biol. https://doi.org/10.1038/nchembio.2007, published online 25 January 2016.

In our original Article, we reported the discovery of a chemical probe for the CBX domains of Polycomb repressive complex 1 (PRC1). In subsequent studies undertaken in our laboratories by Kelsey Lamb (Center for Integrative Chemical Biology and Drug Discovery, Division of Chemical Biology and Medicinal Chemistry, UNC Eshelman School of Pharmacy, University of North Carolina at Chapel Hill, Chapel Hill, NC, USA), we have observed that our modular synthetic approach detailed in this article leads to epimerization at the alanine position. In the reported synthesis in the original paper, the amide coupling of tripeptide intermediate 13 to tripeptide intermediate 7 for the formation of final product UNC3866 (or for the formation of UNC4219, the amide coupling of tripeptide intermediate 13 to tripeptide intermediate 10) resulted in approximately $40 \%$ epimerization at the alanine side chain by NMR. The linear approach outlined in the revised protocol in the Supplementary Note avoids this problem by utilizing a synthetic route in which each amide coupling is performed on the N-Boc-protected amino acid, a method known to diminish epimerization, as validated in our studies by NMR spectra. We note that UNC3866 was originally synthesized using the linear route, despite the modular synthesis reported in the original Supplementary Note, and therefore biological data reported for this compound were not affected. While UNC4219, UNC4007 and UNC4195 were synthesized using the modular route, resynthesis of compounds by the linear route validated that all data obtained using these compounds were unaffected by alanine epimerization. Based on these observations, we provide an alternative synthetic route in this Addendum that avoids alanine epimerization. The protocols have been updated in the Supplementary Note to this Addendum. 